\title{
Polarization Jitters Caused by Fiber Nonlinearity in PM Optical Communication System
}

\author{
Lan-Lan Liu, Chong-Qing Wu* , Wei Yang, Jian Wang, Guo-Dong Liu \\ Institute of Optical Information, School of Science, Beijing Jiaotong University, \\ Key Lab of Education Ministry on Luminescence and Optical Information Technology, Beijing, China \\ Email: ${ }^{*}$ cqwu@bjtu.edu.cn, llliu@bjtu.edu.cn
}

Received 2013

\begin{abstract}
The phenomenon of polarization jitters caused by fiber nonlinearity is investigated. A general formula about the polarization jitter is concluded in polarization multiplexing (PM) system based on two orthogonal linear polarization states when the best polarization correction is used. A $100 \mathrm{~Gb} / \mathrm{s} \mathrm{PM}$ system based on NRZ code is investigated by simulation, and the Stocks parameter about polarization jitter and Poincare sphere diagrams are got for different power and phase difference of two orthogonal polarized light. The results show that the polarization jitters will be suppressed when the combined PM signal is the linear or circular polarization state.
\end{abstract}

Keywords: Optical Fiber Communication; Polarization Multiplexing (PM); Fiber Nonlinearity; Nonlinear Polarization Jitter

\section{Introduction}

In recent years, high-speed optical communication technology has been developed very rapidly. The data rate of single wavelength exceeds $100 \mathrm{~Gb} / \mathrm{s}[1]$, and is headed for $400 \mathrm{~Gb} / \mathrm{s}$. The polarization multiplexing (PM) may double the bit-rate in a single fiber, so it has widely been used in high-speed systems. However, the PM also brought new problems, such as the stability of polarization state, how to keep a good orthogonality in the fiber, how to prevent crosstalk of the two orthogonal signals, etc. In addition, if the two polarization components lose its orthogonality in the transmission process, the demodulation could not be achieved correctly in receivers, the decrease of orthogonality may lead to mutual crosstalk of two polarization components, and the instability of polarization direction could also lead to demodulation error.

The polarization-related effects in the fiber can be divided into linear and nonlinear effect. For linear polarization effect, it is so called the polarization mode dispersion (PMD). After years of research, the method to overcome the influence of linear PMD has been found. At the same time, with the progress of the optical fiber manufacturing technique, linear PMD value is already below, so linear PMD is no longer the key factor.

Another polarization-related effect in the fiber is nonlinear polarization effect. In recent years, the various kinds of multiplexing technologies, such as QPSK, QAM,

\footnotetext{
${ }^{*}$ Corresponding author.
}

WDM, mode division multiplexing, etc., make light power in the fiber increase dramatically, and cause various types of nonlinear effects, including self-phase modulation (SPM), cross phase modulation (XPM), four-wave mixing (FMW), stimulated Brillion scattering (SBS), stimulated Raman scattering (SRS). One of the polarizationrelated nonlinear phenomena, nonlinear polarization cross modulation effect, is the biggest influence of PM. Therefore, to investigate the nonlinear cross polarization modulation and its effect on the transmission is very important.

From the nonlinear mode coupling equation (the nonlinear Schrödinger equation)[5],

$$
\frac{\partial \boldsymbol{A}}{\partial z}+\frac{\mathrm{i}}{2} \frac{\beta_{0}^{\prime \prime}}{T_{0}} \frac{\partial^{2} \boldsymbol{A}}{\partial \tau^{2}}+\frac{\alpha}{2} \boldsymbol{A}=\mathrm{i} \gamma \boldsymbol{G A}
$$

where $\tau=t-\beta_{0}^{\prime} z$ is local time, $\boldsymbol{A}$ is the signal complex amplitude, $\boldsymbol{G}$ is a $2 \times 2$ matrix to describe the characteristics of nonlinear rotation, and is given by

$$
\boldsymbol{G}=\left[\begin{array}{ll}
\left|A_{x}\right|^{2}+\frac{2}{3}\left|A_{y}\right|^{2}, & \frac{1}{3}\left|A_{x}\right|\left|A_{y}\right| \mathrm{e}^{-\mathrm{i} 2 \Delta \phi} \\
\frac{1}{3}\left|A_{x}\right|\left|A_{y}\right| \mathrm{e}^{\mathrm{i} 2 \Delta \phi}, & \left|A_{y}\right|^{2}+\frac{2}{3}\left|A_{x}\right|^{2}
\end{array}\right]
$$

Therefore, research of the nonlinear polarization effect is essentially to investigate the impact of matrix $\boldsymbol{G}$.

The polarization-related nonlinear problem in the fiber has been attracted much more attention. As early as 1986, literature [6] found the unstable phenomenon caused by the nonlinear polarization effect in optical fiber, literature 
[7] supplemented to it. In 1997, D. Marcuse, CR Menyuk, and P. K. A. Wai investigated nonlinear PMD (N-PMD) with random birefringence, and converted the coupled nonlinear Schrödinger equation (CNSE equation) to Manakov-PMD equation [8]. P. K. A. Wai, et al. discussed the N-PMD distributions in different cases [9]. C. R. Menyuk studied the interaction of the Kerr effect and linear PMD [10]. M. Midrio proved that the concept of the principle state of polarization was still correct in $\mathrm{N}$ PMD [11]. B, Crosignani, B. Daino, and P. D. Porto, et al. studied the decreasing in the degree of polarization caused by N-PMD in the low-birefringence fiber[12].

In the PM system, the coupling of the two orthogonal signals resulted from the negative diagonal elements of the matrix $\boldsymbol{G}$ will leads to the crosstalk between two polarization signals, which causes the instability of the two polarization states. This phenomenon is called the random jitter of the polarization state. It was received attention from some writers [13]. However, in the previous literatures, the writers only considered the crosstalk for one channel not for PM. Because the randomicity of two channels is different from one channel, the amplitude, phase even the modulation format are different between the two channels, the jitters will be more serious. Based on the work in [12], this paper will investigate the stability of the orthogonal polarization state and the random jitters of the polarization state for PM system.

In Section 2 of this paper, a general formula to describe the nonlinear polarization rotation in the PM system based on two orthogonal linearly polarized lights is deduced. In section 3 , the jitter of polarization states is corrected by using polarization controllers is investigated. In section 4, simulation experiments about the polarization jitter of the corrected PM system using polarization controllers were performed. The last is the conclusions.

\section{Nonlinear Polarization Rotation of the PM Signal in Optical Fiber}

Generally, a PM system described by a simplified model is shown in Figure 1.

In the figure, a continuous light from LD is divided into two linear polarization light ( $x$ and $y$ ) by the polarization beam splitter (PBS1). And then they are modulated by channel $\mathrm{A}$ and $\mathrm{B}$, the modulation format may be NRZ, DPSK, QPSK, QAM code, and so on. The two linear

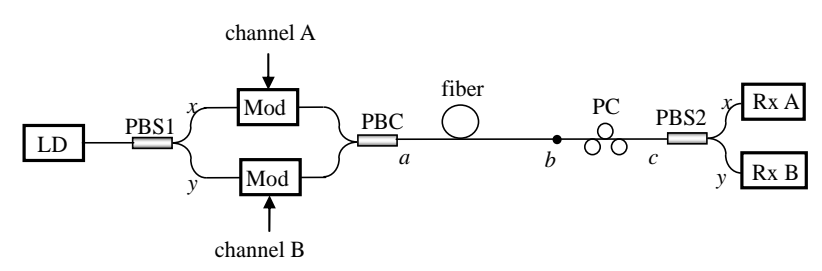

Figure 1. Polarization multiplexing system. polarization light carrying user information will be combined into the multiplexing optical signal (generally speaking, it is elliptically polarized light) in the polarization beam combiner (PBC). When the multiplexing signal is transmitted in optical fiber, its polarization state evolves continuously, and in the output end of fiber it is generally different from the initial polarization state at point $a$. In order to restore the polarization state of point $a$, we should use the polarization controller PC to correct it, and then use polarization beam splitter PBS2 to divide it into $x$ and $y$ polarized light. Finally, in the optical receiver $\mathrm{Rx}$, the modulation code will be received and demodulated. In this figure, the dispersion compensator is not drawing, because the intrinsic dispersion is good compensated.

B. Crosignani, B. Daino, and P. D. Porto, et al in literature [12] presented that in ordinary single-mode fiber with low birefringence, when only considering the nonlinear polarization effect and ignoring the influence of the intrinsic dispersion (because it has been successfully compensated), circularly polarized light could be maintained and nonlinear phase shift could be produced. If $\dot{\phi}_{+}(\tau, z)$ and $\dot{\phi}_{-}(\tau, z)$ represent the complex amplitudes of two orthogonal circularly polarized light in time domain, respectively, we can get

$$
\text { and } \begin{aligned}
\dot{\phi}_{+}(\tau, z) & =T_{+}(\tau) \mathrm{e}^{\mathrm{i} \varphi_{+}(\tau, z)}, \dot{\phi}_{-}(\tau, z)=T_{-}(\tau) \mathrm{e}^{\mathrm{i} \varphi_{-}(\tau, z)} \\
\varphi_{+}(\tau, z) & =\gamma \frac{2}{3}\left[\left|T_{+}(\tau)\right|^{2}+2\left|T_{-}(\tau)\right|^{2}\right] z+\varphi_{0_{+}}(\tau) \\
\varphi_{-}(\tau, z) & =\gamma \frac{2}{3}\left[2\left|T_{+}(\tau)\right|^{2}+\left|T_{-}(\tau)\right|^{2}\right] z+\varphi_{0_{-}}(\tau)
\end{aligned}
$$

But in practical PM system, two multiplexing polarized lights are linear, so we first change the equations into the evolution of linear polarized light.

Assume the complex vectors of two orthogonal lights at $z=0$ (point $a$ ) are as following,

$$
\boldsymbol{A}(\tau, 0)=\left[\begin{array}{c}
\dot{A}_{x}(\tau, 0) \\
0
\end{array}\right] \text {, and } \boldsymbol{B}(\tau, 0)=\left[\begin{array}{c}
0 \\
\dot{B}_{y}(\tau, 0)
\end{array}\right]
$$

where $\dot{A}_{x}(\tau, 0)=A_{x 0} \mathrm{e}^{\mathrm{i} \varphi_{x 0}}, \dot{B}_{y}(\tau, 0)=B_{y 0} \mathrm{e}^{\mathrm{i} \varphi_{y 0}}$, and $A_{x 0}=A_{x 0}(\tau)$, $\varphi_{x 0}=\varphi_{x 0}(\tau), B_{y 0}=B_{y 0}(\tau), \varphi_{y 0}=\varphi_{y 0}(\tau)$, they are all real and a function of time, carrying user information. Then, after multiplexer PBC, the output light is given by $\boldsymbol{C}(\tau, 0)=\left[\begin{array}{ll}\dot{A}_{x}(\tau, 0) & \dot{B}_{y}(\tau, 0)\end{array}\right]^{\mathrm{T}}$, the relationship between the complex amplitudes of circularly polarized light and linearly polarized light in the time domain is

$$
\dot{\phi}_{+}=(\sqrt{2} / 2)\left(\dot{A}_{x}-\mathrm{i} \dot{B}_{y}\right), \dot{\phi}_{-}=(\sqrt{2} / 2)\left(\dot{A}_{x}+\mathrm{i} \dot{B}_{y}\right)
$$

After performing very complicated mathematical operation, and considering the fiber loss (the coefficient is $\alpha$ ), we can get the complex vector of the optical signal transmitted in the fiber at point $b$ 


$$
\left[\begin{array}{c}
\dot{C}_{x}(\tau, z) \\
\dot{C}_{y}(\tau, z)
\end{array}\right]=\mathrm{e}^{\mathrm{i} \gamma z\left(A_{x 0}^{2}+A_{y 0}^{2}\right)-\frac{\alpha z}{2}}\left[\begin{array}{cc}
\cos \theta & \sin \theta \\
-\sin \theta & \cos \theta
\end{array}\right]\left[\begin{array}{c}
\dot{A}_{x}(\tau, 0) \\
\dot{B}_{y}(\tau, 0)
\end{array}\right]
$$

where $z_{\text {eff }}=\left(1-\mathrm{e}^{-\alpha z}\right) / \alpha$ is the fiber nonlinear effective length, and $\theta(\tau)=(2 / 3) \gamma z_{\text {eff }} A_{x 0} B_{y 0} \sin \Delta \varphi_{0}$.

From formula (8), we can see that due to the nonlinear polarization effect, the polarization rotation will occur, but the orthogonality of two signals can still be maintained. The rotating angle $\theta(\tau)$ is related to the fiber nonlinear coefficient $\gamma$, nonlinear effective length $z_{\text {eff }}$, amplitudes of two multiplexing signals $A_{x 0}(\tau)$ and $B_{y 0}(\tau)$, phase difference $\Delta \varphi_{0}$, and etc. Because $A_{x 0}(\tau)$ and $B_{y 0}(\tau)$ are all random variable, polarization will jitter.

\section{Polarization Jitters after Polarization State Correction}

As stated above, in order to perform polarization demultiplexing correctly, we must correct the polarization state using a polarization controller (see Figure 1). Compared with the speed of change of the modulation signal, the adjusting speed of the polarization controller is very low, so only the low order correction can be realized. We only consider the mean value correction, it means that the polarization controller only corrects the mean value of the nonlinear polarization rotation angle.

Assume the corrective angle produced by the polarization controller is $\bar{\theta}$, which should be the mean value of the nonlinear rotation angle $\theta(\tau)$, i.e.

$$
\bar{\theta}=<\theta(\tau)>=\frac{2}{3 T} \gamma Z_{\text {eff }} \int_{0}^{T} A_{x 0}(\tau) B_{y 0}(\tau) \sin \Delta \varphi_{0}(\tau) \mathrm{d} \tau
$$

where $T$ is the action time. So, the compensation matrix of $\mathrm{PC}$ is given by

$$
\boldsymbol{U}_{\mathrm{PC}}=\left[\begin{array}{cc}
\cos \bar{\theta} & -\sin \bar{\theta} \\
\sin \bar{\theta} & \cos \bar{\theta}
\end{array}\right]
$$

It is not difficult to find out the polarization state of corrected light at point $c$ is

$$
\left[\begin{array}{c}
\dot{C}_{x}(\tau, z) \\
\dot{C}_{y}(\tau, z)
\end{array}\right]=\mathrm{e}^{\mathrm{i} \gamma z\left(A_{x 0}^{2}+B_{y 0}^{2}\right)-\frac{\alpha z}{2}} U_{\mathrm{PC}}\left[\begin{array}{cc}
\cos \theta & \sin \theta \\
-\sin \theta & \cos \theta
\end{array}\right]\left[\begin{array}{c}
\dot{A}_{x}(\tau, 0) \\
\dot{B}_{y}(\tau, 0)
\end{array}\right]
$$

To substitute formula (10) into (11), we can get

$$
\left[\begin{array}{c}
\dot{C}_{x}(\tau, z) \\
\dot{C}_{y}(\tau, z)
\end{array}\right]=\mathrm{e}^{\mathrm{i} \gamma z\left(A_{x 0}^{2}+B_{y 0}^{2}\right)-\frac{\alpha z}{2}}\left[\begin{array}{cc}
\cos \Delta \theta & -\sin \Delta \theta \\
\sin \Delta \theta & \cos \Delta \theta
\end{array}\right]\left[\begin{array}{l}
\dot{A}_{x}(\tau, 0) \\
\dot{B}_{y}(\tau, 0)
\end{array}\right]
$$

Generally, $\Delta \theta(\tau)=\bar{\theta}-\theta(\tau) \neq 0$, the jitter of polarization state will be observed. From Equation. (12) we can get the instantaneous Stocks parameters

$$
S_{0}(\tau, z)=\mathrm{e}^{-\alpha z}\left[P_{A 0}(\tau)+P_{B 0}(\tau)\right]
$$

$$
\begin{gathered}
S_{1}(\tau, z)=\mathrm{e}^{-\alpha z}\left\{\cos 2 \Delta \theta\left[P_{A 0}(\tau)-P_{B 0}(\tau)\right]\right. \\
\left.-2 \sin 2 \Delta \theta \sqrt{P_{A 0}(\tau) P_{B 0}(\tau)} \cos \Delta \varphi\right\} \\
S_{2}=\mathrm{e}^{-\alpha z}\left\{\sin 2 \Delta \theta(\tau)\left[P_{A 0}(\tau)-P_{B 0}(\tau)\right]\right. \\
\left.+2 \cos 2 \Delta \theta(\tau) \sqrt{P_{A 0}(\tau) P_{B 0}(\tau)} \cos \Delta \varphi_{0}\right\} \\
S_{3}=\mathrm{e}^{-\alpha z} 2 \sqrt{P_{A 0}(\tau) P_{B 0}(\tau)} \sin \Delta \varphi_{0}
\end{gathered}
$$

In Equation (13)-(16), the $P_{A 0}(\tau)$ and $P_{B 0}(\tau)$ are the initial power of channel A and B, respectively. In order to draw the Poincare sphere, we should normized Stocks parameters, which are described as following

$$
\begin{aligned}
S_{0}(\tau, z)= & 1 \\
S_{1}(\tau, z)= & {\left[\cos 2 \Delta \theta\left[P_{A 0}(\tau)-P_{B 0}(\tau)\right]\right.} \\
& -\frac{\left.2 \sin 2 \Delta \theta \sqrt{P_{A 0}(\tau) P_{B 0}(\tau)} \cos \Delta \varphi\right]}{\left[P_{A 0}(\tau)+P_{B 0}(\tau)\right]} \\
S_{2}(\tau, z)= & {\left[\sin 2 \Delta \theta(\tau)\left[P_{A 0}(\tau)-P_{B 0}(\tau)\right]\right.} \\
& +\frac{\left.2 \cos 2 \Delta \theta(\tau) \sqrt{P_{A 0}(\tau) P_{B 0}(\tau)} \cos \Delta \varphi_{0}\right]}{\left[P_{A 0}(\tau)+P_{B 0}(\tau)\right]} \\
S_{3}(\tau, z)= & \frac{2 \sqrt{P_{A 0}(\tau) P_{B 0}(\tau)} \sin \Delta \varphi_{0}}{\left[P_{A 0}(\tau)+P_{B 0}(\tau)\right]}
\end{aligned}
$$

\section{Simulation Experiment of the Polarization Jitters for NRZ Code}

Although NRZ code has rarely been used in high speed optical communication system, as a kind of basic modulation format, its research is still meaningful. The bit-rate of PM system is $100 \mathrm{~Gb} / \mathrm{s}$. For these high speed systems, we generally use a narrow linewidth laser smaller than $100 \mathrm{kHz}$ with excellent coherence performance, and the coherence time is larger than $10 \mu \mathrm{s}$. So for a $100 \mathrm{~Gb} / \mathrm{s}$ signal with 10ps pulse width, the phase difference $\Delta \varphi_{0}$ between two multiplexing signals can be thought of a constant in 1000 bits. The simulation is divided into two cases, one is the power of the two multiplexing signals are equal, all are $10 \mathrm{mw}$, so that the circular polarization state may appear; the other one is to assume the power of interferencing signal is two times of the interferenced signal, namely channel A is $10 \mathrm{mw}$, channel B is $20 \mathrm{mw}$, so the circular polarization state does not exist. When simulating, the initial signal-to-noise ratio (SNR) of NRZ code is assumed to be $30 \mathrm{~dB}$, which is a good signal. The optical fiber length is $50 \mathrm{~km}$, and the loss is $0.2 \mathrm{~dB} / \mathrm{km}$, the effective nonlinear length is $19.54 \mathrm{~km}$. Figure 2 shows 100 bit waveform (left) and eye diagram (right) of input signal (channel A).

\subsection{Polarization Multiplexing with Equal Power}

In the first case, the power of the two multiplexing sig- 
nals is equal. Figures 3-6 shows the evolution of the polarization states of output combined multiplexed signal at point $c$ in different initial phase difference $\Delta \varphi_{0}$, respectively. Left figures are Stocks parameters with 100 bits, and right figures are Poincare sphere diagrams. In Figure 3, $\Delta \varphi_{0}=0$, the combined multiplexed signal is linearly polarized light. In Figures 4-6, $\Delta \varphi_{0}=\pi / 8, \pi / 4$, and $3 \pi / 8$, respectively, the combined multiplexed signal is elliptically polarized The figures show that when the

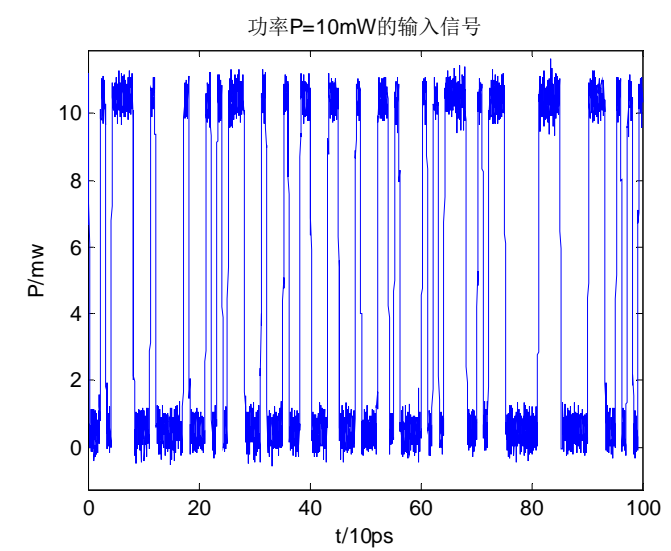

combined multiplexed signals are linearly and circularly polarized light, there is no obvious polarization jitter, the evolution of polarization states is only along with the Equator; when the combined multiplexed signals are elliptically polarized lights, there is a obvious polarization jitter, the polarization states will be scattering, and the degree of polarization (DOP) will decrease. The strong polarization jitter occurs when $\Delta \varphi_{0}=\pi / 4$ (see Figure 5). It makes the difficulty for demultiplexing by these jitters.

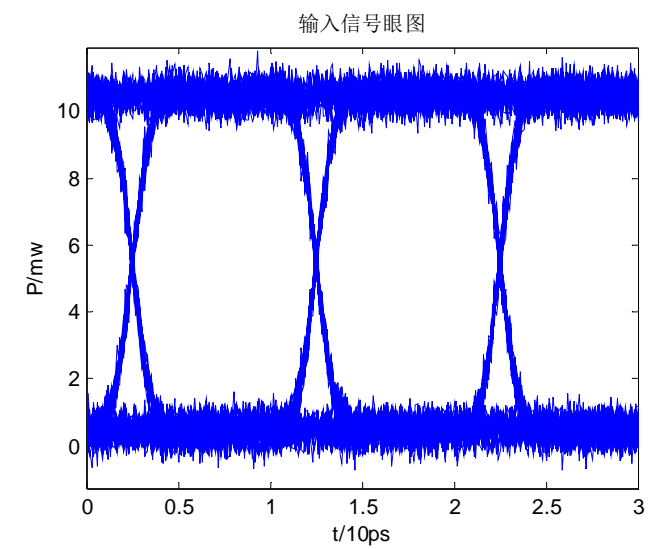

Figure 2. The waveform (left) and eye diagram (right)of the input signal.
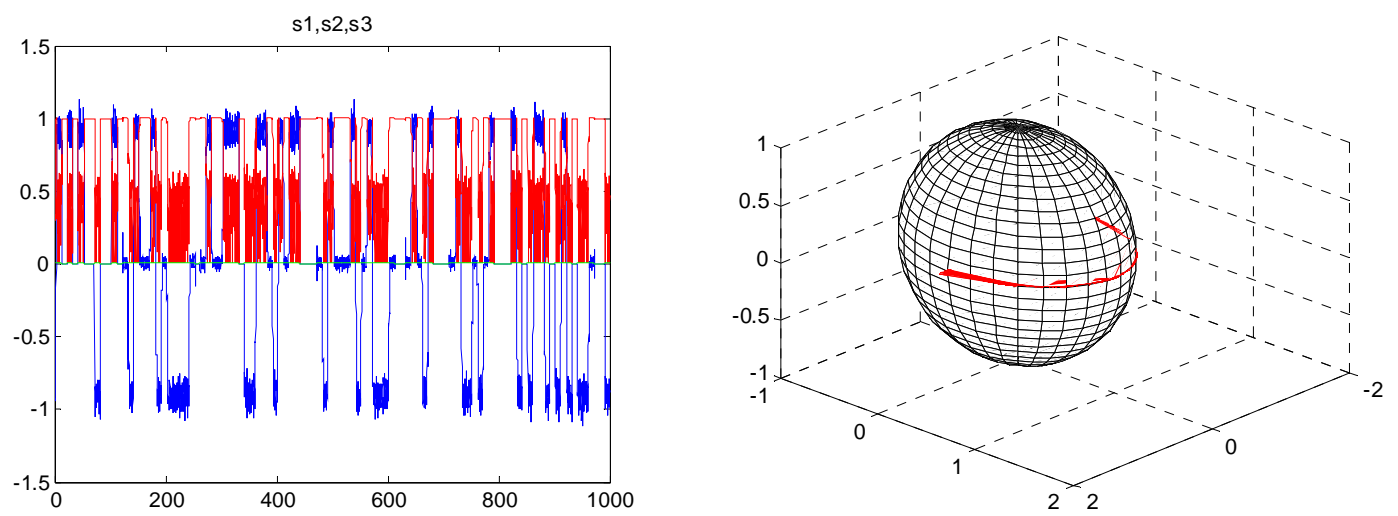

Figure 3. The Stocks parameters (left) and Poincare sphere diagram (right) of the output signal when $\Delta \varphi_{0}=0$.
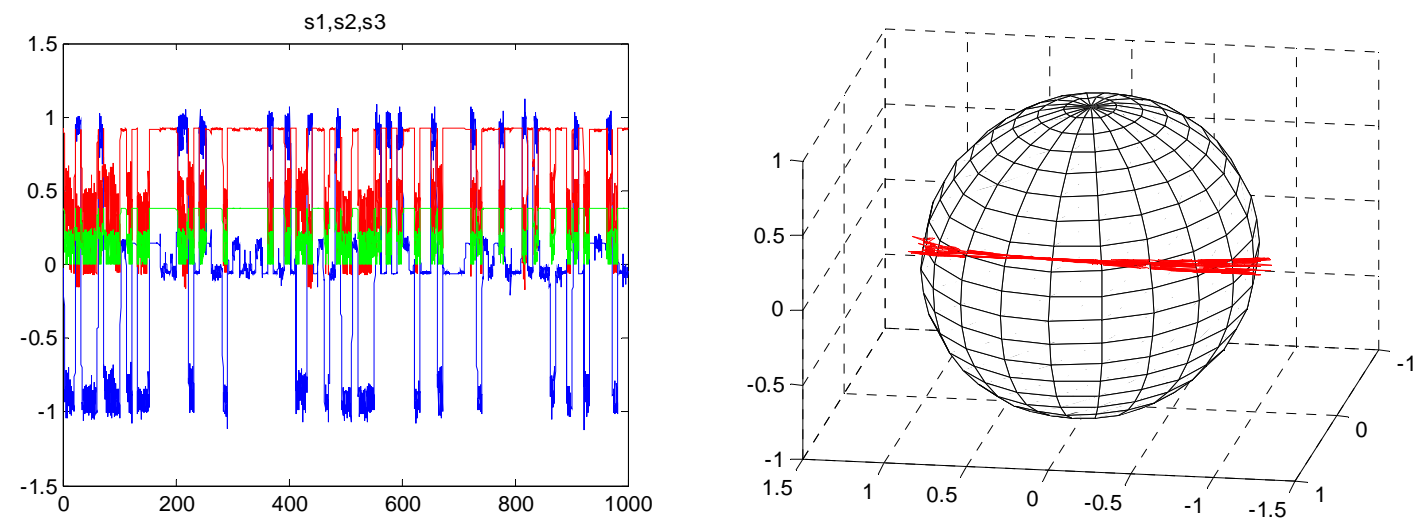

Figure 4. The Stocks parameters (left) and Poincare sphere diagram (right) of the output signal when $\Delta \varphi_{0}=\pi / 8$. 

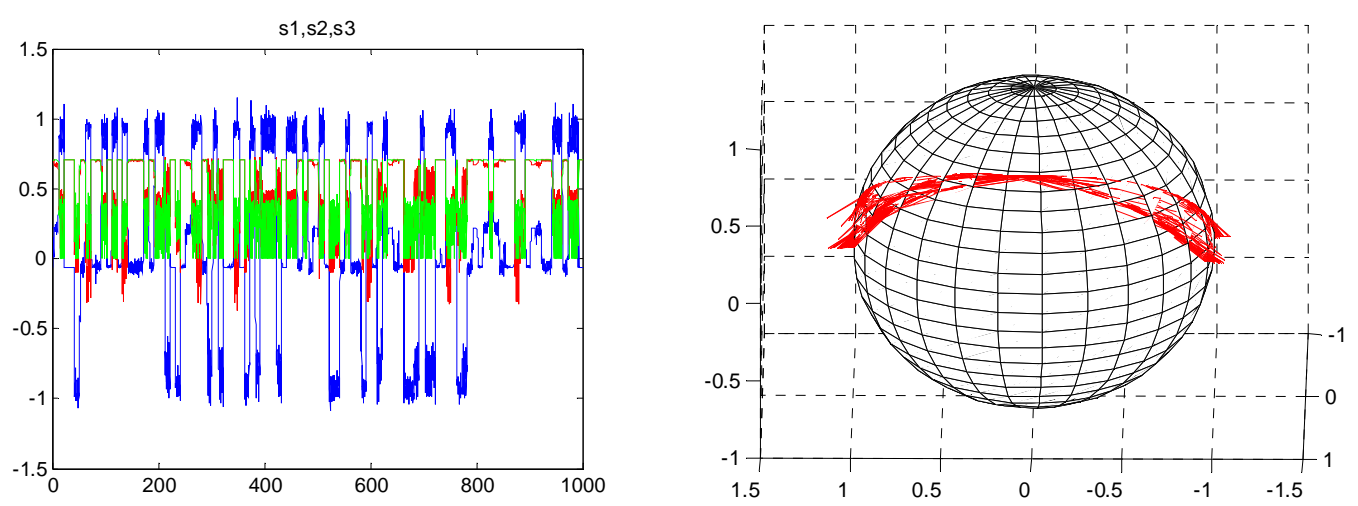

Figure 5. The Stocks parameters (left) and Poincare sphere diagram (right) of the output signal when $\Delta \varphi_{0}=\pi / 4$.
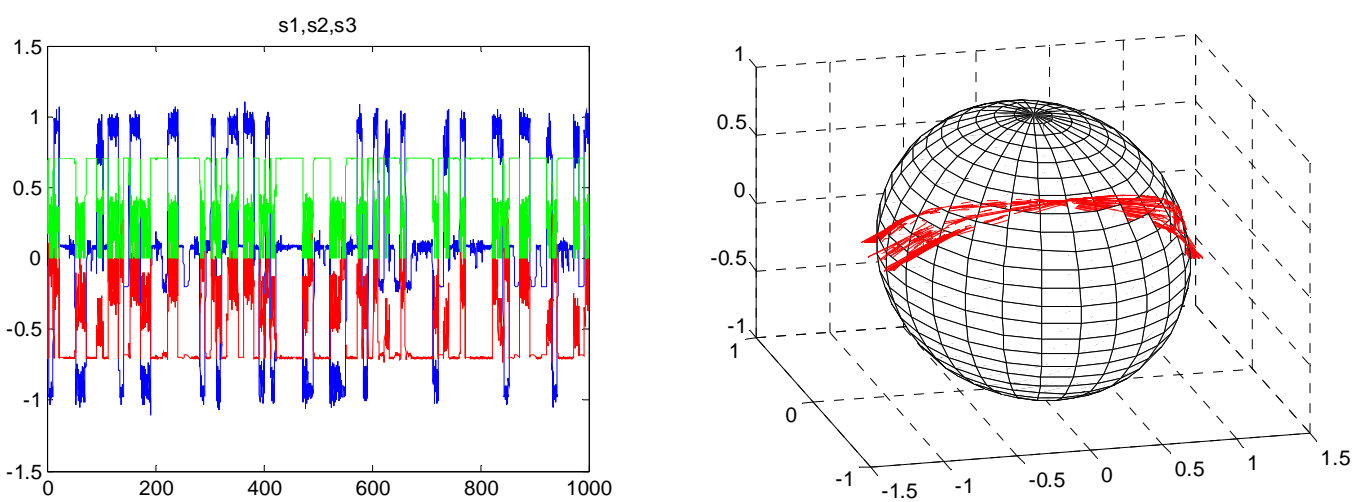

Figure 6. The Stocks parameters (left) and Poincare sphere diagram (right) of the output signal when $\Delta \varphi_{0}=3 \pi / 8$.
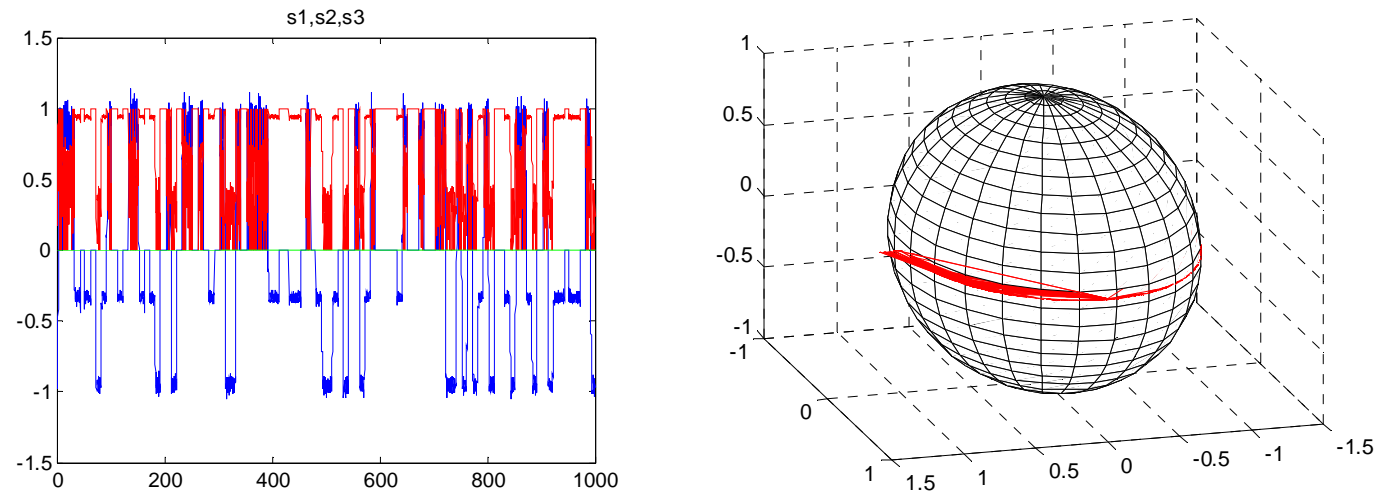

Figure 7. The Stocks parameters (left) and Poincare sphere diagram (right) of the output signal when $\Delta \varphi_{0}=0$.

\subsection{Polarization Multiplexing with Unequal Power}

In this case, the power of channel $\mathrm{A}$ is $10 \mathrm{~mW}$, and the power of channel B is 20mW. Figures 7-11 shows the evolution of the polarization states of output combined multiplexed signal at point $c$ in different initial phase difference $\Delta \varphi_{0}$, respectively. Left figures are stocks parameters with 100 bits, and right figures are Poincare sphere diagrams. In Figure 7, $\Delta \varphi_{0}=0$, the combined multiplexed signal is linearly polarized light. In Figures
8-11, $\Delta \varphi_{0}=\pi / 8, \pi / 4, \pi / 2$ and $3 \pi / 4$, respectively, the combined multiplexed signal is elliptically polarized. The figures show that when the combined multiplexed signals are linearly, there is no obvious polarization jitter, the evolution of polarization states is only along with the Equator; when the combined multiplexed signals are elliptically polarized, there is obvious polarization jitter, the polarization states will be scattering and the degree of polarization (DOP) will decrease. The strong polarization jitter occurs when $\Delta \varphi_{0}=\pi / 2$ 
(see Figure 10). It makes it difficult for demultiplexing by these jitters.

\subsection{Discussion and Suggestions}

Compared Figures 3-6 with Figures 7-11, we can draw two important conclusions:

(1) PM with unequal optical power can cause much stronger jitter than that with equal power.

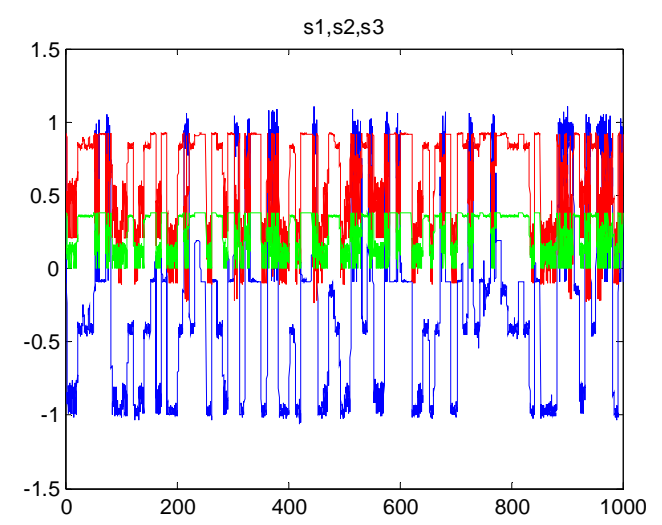

(2) When the combined multiplexed light is linear or circular polarization state, there is no jitters basically, i.e. when the phase difference between two modulation arms is 0 or $\pi$, or $\Delta \varphi_{0}=\pi / 2$ in the case of equal power, polarization noise can be suppressed very well.

According to the above solution, the power of two channels should be equal and the initial phase difference should be 0 or $\pi$ to reduce the polarization jitter.

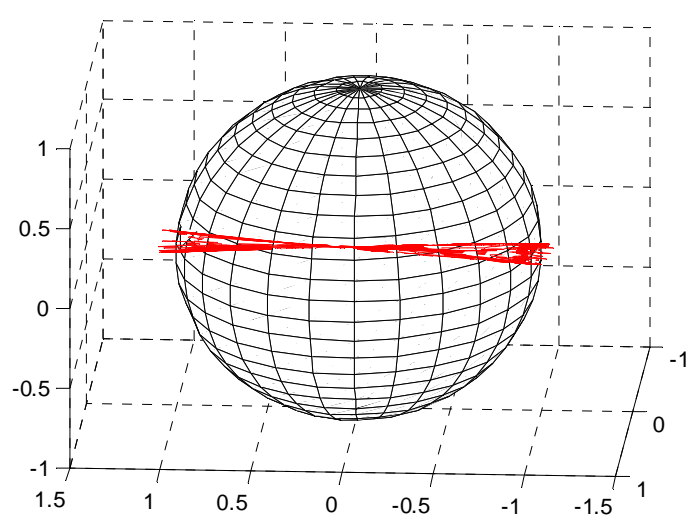

Figure 8. The Stocks parameters (left) and Poincare sphere diagram (right) of the output signal when $\Delta \varphi_{0}=\pi / 8$.
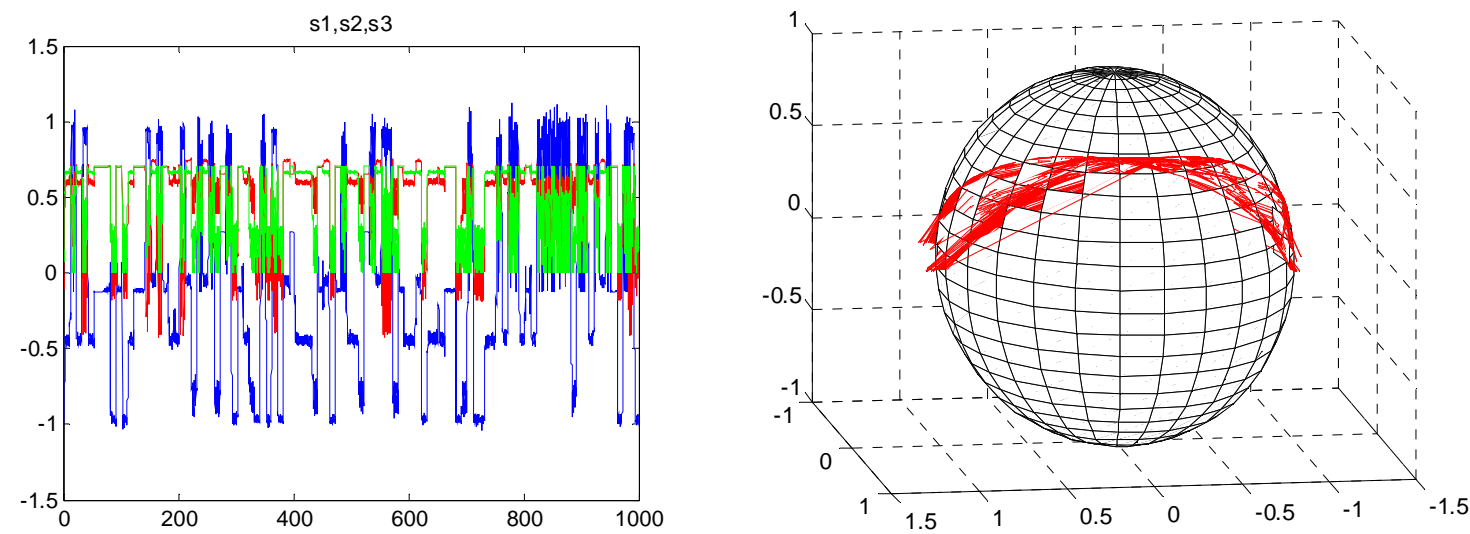

Figure 9. The Stocks parameters (left) and Poincare sphere diagram (right) of the output signal when $\Delta \varphi_{0}=\pi / 4$.
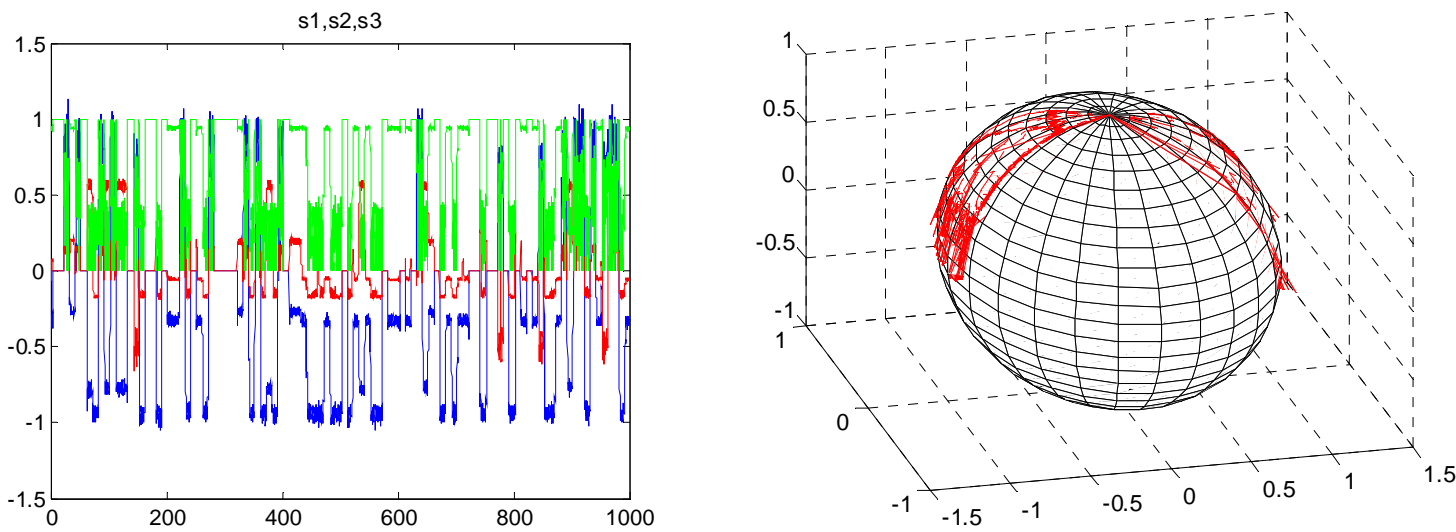

Figure 10. The Stocks parameters (left) and Poincare sphere diagram (right) of the output signal when $\Delta \varphi_{0}=\pi / 2$. 

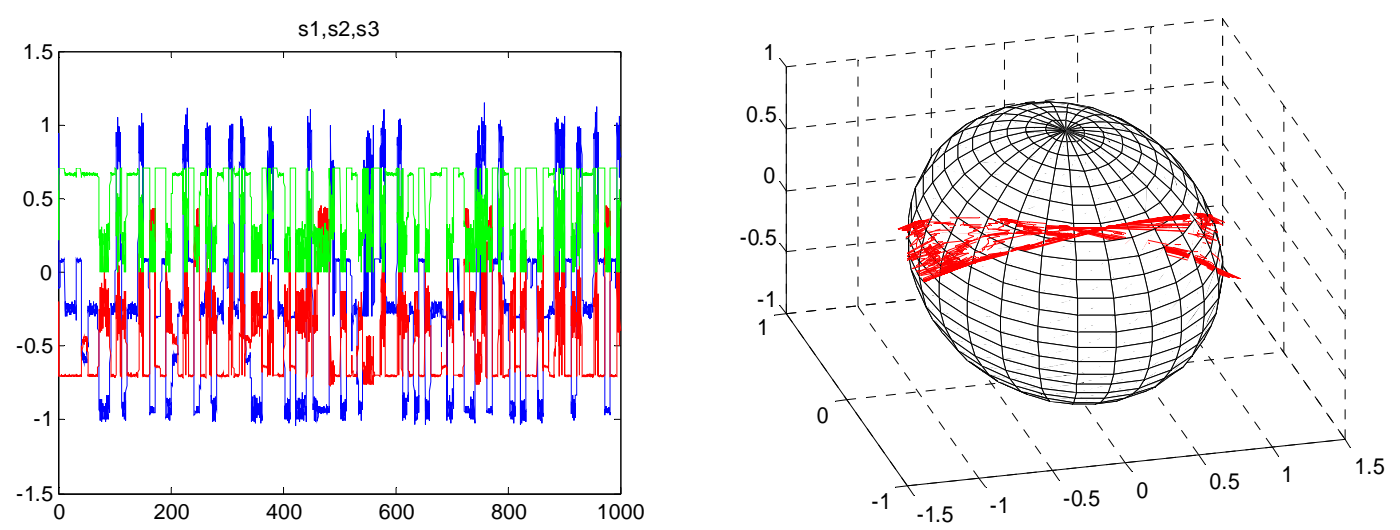

Figure 11. The Stocks parameters (left) and Poincare sphere diagram (right) of the output signal when $\Delta \varphi_{0}=3 \pi / 4$.

\section{Conclusions}

The polarization jitter resulted from fiber nonlinear polarization modulation is thoroughly investigated. The polarization jitter in the case of the best polarization correction is discussed, a general formula about jitters is concluded. The results show that the jitters are depending on the power of two multiplexing light, initial phase difference, nonlinear coefficient, effective nonlinear length, and etc. A $100 \mathrm{~Gb} / \mathrm{s}$ PM system based on NRZ code is investigated by simulation, the evolutions of the polarization states and Stocks parameters of output combined multiplexed signal are got in different initial phase difference $\Delta \varphi_{0}$, respectively. In order to reduce the polarization jitter, the power of two channels should be equal and the initial phase difference should be 0 or $\pi$. The above conclusions have important guiding significance for large capacity, high speed optical communication system.

\section{Acknowledgements}

This paper thanks the support of National Nature Science Foundation of China, under grant 61275075, 61077048, and National Nature Science Foundation of Beijing, under grant 4112042.

\section{REFERENCES}

[1] Satoki Kawanishi, "100Gb/s Optical Communication Systems”, OFC' 2011, WH5.

[2] S. Ferber, R. Ludwig, C. Boerner, et al, “160 Gb/s DPSK Transmission over $320 \mathrm{~km}$ Fibre Link with High Long-Term Stability,” Electronics Letters, 17th February 2005 Vol. 41 No. 4.doi:10.1049/el:20057179

[3] P. Bousselet, H. Bissessur, J. Lestrade, et al, "High Capacity (64 x $43 \mathrm{~Gb} / \mathrm{s}$ ) Unrepeatered Transmission over 440 km”, OFC’2011, OMI2.

[4] D. Foursa, Y. Cai, et al, "Coherent 40 Gb/s Transmission with High Spectral Efficiency Over Transpacific Distance,” OFC'2011, OMI4,

[5] G. Agrawal, "Nonlinear Fiber Optics and Applications on nonlinear Fiber Optics,” 3rd Edition.

[6] H. G. Winful, "Polarization Instabilities in Birefringence Nonlinear Media: Application for Fiber-Optic Devices. Optical Letters, 1986,Vol.11, No.1,pp.33-35. doi:10.1364/OL.11.000033

[7] C.Q. Wu, Y. Tao, L. LI, Y. J. Wang and X.J. Xin, "Investigation on Nonlinear Polarization Instability in Optical Fibers,” Semiconductor Optoelectronics, Vol.20, No.5, 1999.

[8] D. Marcuse, C. R. Menyuk and P. K. A. Wai, "Application of the Manakov-PMD Equation to Studies of Signal Propagation in Optical Fibers with Randomly Varying Birefringence,” Journal of Lightwave Technology, Vol. 15, No. 9, 1997, pp. 1735-1746.doi:10.1109/50.622902

[9] P. K. A. Wai, W. L. Kath, C. R. Menyuk, et al, "Nonlinear Polarization-Mode Dispersion in Optical Fibers with Randomly Varying Birefringence, JOSA. B, Vol. 14, No. 11, p.2967, 1997.

[10] C. R. Menyuk and B. S. Marks, "Interaction of Polarization Mode Dispersion and Nonlinearity in Optical Fiber Transmission Systems," Journal of Lightwave Technology, Vol. 24, No. 7, 2006, pp. 2806-2826. doi:10.1109/JLT.2006.875953

[11] M. Midrio, "Nonlinear Principal States of Polarization in Optical Fibers with Randomly Varying Birefringence," JOSA B, Vol.17, No.2, pp.169-177, 2000. doi:10.1364/JOSAB.17.000169

[12] B. Crosignani, B. Daino and P. D. Porto, "Depolarization of Light due to the Optical Kerr Effect in Low-Birefringence Single-Mode Fibers," JOSA B, Vol. 3, No. 8, 1986, pp. 1120-1123. doi:10.1364/JOSAB.3.001120

[13] C.Q. Wu, "Polarization Rotation of Pump Light in Raman Fiber Amplifier with Attenuation,” Journal of Beijing Jiaotong University, Vol.33, No.6, 2009, pp.1-8. 